\title{
Research Hotspots and Evolution of Fusion of Blockchain and Supply Chain in China Based on Visualization Analysis
}

\author{
Xuedong Liang ${ }^{1,2}$, and Jinghong Sun ${ }^{1 *}$ \\ ${ }^{1}$ Business School, Sichuan University, Chengdu, Sichuan,610000, China \\ ${ }^{2}$ Sichuan Haina Rendong Science and Technology Co. Ltd.
}

\begin{abstract}
Based on 837 literatures of CNKI from 2015 to 2020, this paper conducts visualization analysis about domestic fusion of blockchain and supply chain with the utilization of Citespace, and reveal the research hotspots in the field of fusion of blockchain and supply chain in the past five years. The study finds that, firstly, China Finance, Southwest Finance, and Business \& Economy are the journals with higher quantity of publication in this field; secondly, Song Hua and Xu Peng are the leading scholars in this research area; thirdly, the research institutions in this field are mainly concentrated in the School of Economics and Management, Law School and School of Information Management in major universities; fourthly, application scenarios, technology development, applied agent and fund are the research hotspots in this area. This study reveals the basic dynamics of domestic fusion of blockchain and supply chain research in the past five years, which not only can promote the theoretical development of this field, but also point out the research hotspots and future research direction for scholars in this area, and provide reference and reference for all walks of life who plans to enter this field.
\end{abstract}

\section{Introduction}

In recent years, blockchain and supply chain have been developing rapidly, but the blocked information flow, difficult transaction disputes, and the lack of trust between the parties often occur. A large number of scholars have conducted in-depth research on how to solve these problems and found that the blockchain and supply chain have a natural "dual-chain integration" mechanism that can effectively solve a series of problems. In 2016, Liu Dacheng of Tsinghua University put forward the concept of "dual-chain integration" for the first time, that is, "blockchain + supply chain". We can solve the pain points of the supply chain through the advantages of block chain and use the strengths of the supply chain to make up for the shortcomings of block chain to achieve effective fusion.

Domestic scholars have conducted in-depth research on issues related to blockchain and supply chain. In the supply chain field, Zhao Kan et al. (2017) used word frequency analysis method and Note-Express to summarize the research focus of domestic scholars in supply chain management in the past ten year ${ }^{[1]}$,Xue Yanxiao (2020) used CiteSpace to analyse the domestic supply chain management research literature from 2010 to 2020, and obtained the research hotspots of the supply chain and the future trend of the supply chain research ${ }^{[2]}$. Aiming at the field of blockchain, Zhai Tong and $\mathrm{Li}$
Wenlan (2019) made analysis on the Chinese patent literature of blockchain technology, and obtained the research hotspots and current status of the domestic blockchain industry ${ }^{[3]}$. Regarding the dual-chain fusion field, Wang Junyu (2019) analysed the current research status of the domestic blockchain field from five aspects including finance, medical care, energy Internet, supply chain, and education ${ }^{[4]}$; Li Xudong (2019) summarized the research direction of application of blockchain in the field of integrated logistics, partial logistics and supply chain logistics [5]; Yi Qiang et al. (2020) analysed the application status of block chain technology in fields of cross-border e-commerce, supply chain, and intelligent logistics ${ }^{[6]}$.

It can be seen from the above studies that domestic scholars have conducted a lot of exploration in the field of supply chain and blockchain, and have obtained rich research results. However, it can be seen from the existing literature that the current research has certain shortcomings: firstly, systematic research on the "hot spots of this field and its evolution" is lacking. This type of research can reflect the focus of dual-chain fusion research and promote the development of related theories of dual-chain fusion. Secondly, the analysis of literature data is not detailed enough. In view of this, this paper studies and analyses the domestic research literature on blockchain and supply chain from 2015 to 2020 in the "CNKI" by using the co-occurrence network analysis method. 


\section{Research design}

\subsection{Research method}

Based on the mixed research method of bibliometrics, this study summarizes the past research hotspots and evolution process of domestic dual-chain fusion, and predicts the future development trend. The specific research process is as follows: first, this article analyses the core author, journal and institution in the domestic research field of dual-chain fusion based on the Citespace software, presenting the basic situation of research of this field; secondly, it analyses the domestic research literature of the domestic research based on the co-occurrence analysis method; then it further demonstrates the current domestic research subdivision areas of the dual-chain fusion, which will help us to further deepen our understanding of the structure, law and distribution of the scientific knowledge, and explores the development of the discipline knowledge field and its research hotspots, frontiers and trend.

\subsection{Data collection}

This study focuses on the structure and evolution of domestic dual-chain fusion research, and the sample literature comes from the "CNKI". First, we clarified that domestic research on this field started in 2015 through a preliminary search; then, we set the retrieval time as 2015 2020, "blockchain + supply chain" and "dual-chain fusion" as keywords, and conducted multiple retrieval with "topic"; additionally, we set the sources of journal as "CSSCI Journal Sources", "CSCD Journal Sources" and "Peking University Core Journal Sources", excluding irrelevant literatures. After statistics, we obtain 837 valid literatures.

\section{Basic statistical analysis of domestic dual-chain fusion}

\subsection{Journal}

Through the statistic, we find that the journals with higher publication volume in this field are: China Finance, Southwest Finance, Journal of Commercial Economics, China Business and Market, Construction Economy, Computer Engineering and Applications, and Computer Engineering. Among them, China Finance, Southwest Finance, and Journal of Commercial Economics have the highest number of publications with 10 articles each, accounting for $1.19 \%$ respectively. And we come to a conclusion that there is no centralized journal cluster in the research of this field, and the research on it is currently in a situation of interdisciplinary and diverse research topics. In addition, these journals also show different theme representations and content biases. For example, the main content of the literature on this field in China Finance, and Southwest Finance involves the application and research of blockchain in supply chain finance; Journal of Commercial Economics focuses more on the application mode of supply chain based on blockchain technology; China Business and Market is biased towards the optimization of supply chain management under the blockchain technology; the main content of Construction Economy is the research on the construction supply chain information management system under blockchain technology; Computer Engineering and Applications, and Computer Engineering tends to study the development of blockchain and supply chain technology as well as the supply chain traceability system based on blockchain

\subsection{Author}

Through the statistic of prolific authors on domestic dualchain fusion research, we find that among the high-yield authors of domestic dual-chain research, there are 21 authors with more than 3 papers published. And it should be pointed out that Song Hua and Xu Peng are at the center of the high-yield authors, who are the leading scholars in the study of dual-chain fusion with 19 and 11 papers respectively. And we conclude that scholars in this field lack communication.

\subsection{Institution}

Through the statistical analysis, we find that the top 15 institutions published 125 papers, accounting for $14.93 \%$. Among them, Renmin Business School issued 22 articles, accounting for $2.63 \%$; Renmin University China Law School issued 14 articles, accounting for $1.67 \%$; School of Economics and Management of Taiyuan University of Science and Technology published 11 articles, accounting for $1.31 \%$; Antai College of Economics and Management of Shanghai Jiao Tong University and School of Management Tianjin University both issued 10 articles, accounting for $1.19 \%$. At the same time, we draw a conclusion that the research institutions in this field are mainly concentrated in the School of Economics and Management, Law school, and Information Management School in major universities.

\section{Visualization analysis of domestic research on dual-chain fusion}

\subsection{Visualization analysis of research hotspot identification based on keyword co-occurrence}

Through statistical analysis, the results show that there are 36 keywords with frequency greater than or equal to 5 . The top 20 keywords, in order, are: block chain, supply chain finance, block chain technology, funding constraints, supply chain, digital currency, intelligent contracts, financial technology, supply chain financing, online finance, decentration, artificial intelligence, the precision of poverty alleviation, big data, credit risk, the small and medium-sized enterprises, supply chain coordination, legal digital currency, regulatory sandbox, financial regulation, the corresponding frequency are:340, 185, 79, $53,41,39,39,36,28,25,22,19,19,17,17,17,17,16$, 15,13 . Based on the above co-occurrence keywords, this paper further summarized the hot topics in the field: 
supply chain finance, supply chain collaborative management, and food safety traceability. The internal logic of the above themes is as follows: supply chain finance solves the financing problems of enterprises, supply chain collaborative management highlights the comparative advantages of dual-chain fusion, food safety traceability is the main application scenario of dual-chain fusion.

\subsubsection{Supply Chain Finance. Finance has always been an} important application field of dual-chain fusion applications. At present, some scholars have explored the research of dual-chain fusion in financial scenarios, which can be divided into the following two aspects:

(1) Research on the application model of supply chain finance

At present, the rapid development of Internet technology provides powerful technical support and application scenarios for supply chain financial services. It is a development trend of the financial industry for Internet companies and traditional financial companies to cooperate and develop together.

Regarding the supply chain financial model, scholars have conducted research in recent years from the application model of the Internet supply chain finance era. Shi Jinzhao and Guo Jue (2015) investigated whether the banking business includes e-commerce and divided the supply chain finance into three patterns based on the bilateral interactive perspective of bank and e-commerce platform. ${ }^{[7]}$ Zhuang Lei et al. (2019) analyzed the model of blockchain technology embedded in the financial industry and proposed the development path of applying block chain technology to drive financial business innovation $^{[8]}$. Deng Aimin et al. (2019) conducted a coupling analysis of blockchain technology and supply chain factoring.

(2) Research on the financing model of supply chain finance ${ }^{[9]}$.

There are generally four participants in supply chain financial activities, so there are four sources of funds in the financing process of supply chain finance, and these four financing modes of fund supply also have different characteristics. Specifically: financial institutions dominated by banks generally have sufficient capital reserves and more professional risk management technology; e-commerce platforms can implement precise financing for companies and monitor subsequent risks by virtue of their absolute data and information advantages; supplier participants have an absolute advantage in improving the efficiency of the supply chain; finally, because logistics provider is embedded in all links of the supply chain, logistics providers can carry out accurate value evaluation of goods.

First, financial institutions dominated by mainly banks act as capital providers. Chen Yonghui et al. (2018) designed a trade credit financing model based on the differences among retailers in the supply chain and studied the role of the differences of retailers in determining the supply chain financial model of the core enterprises ${ }^{[10]}$. Second, e-commerce platforms act as the provider, $\mathrm{Tu}$ Jianping et al. (2013) studied and compared four models of supply chain finance under the background of e- commerce platforms and traditional financing models, then designed an e-commerce platform performance evaluation index system [11]. Furthermore, the direct participants in the supply chain are the providers. Wang Mingzheng et al. (2017) discussed the supply chain coordination problem when retailers may default and they are in a competitive state on the background of trade credit between several retailers and one supplier ${ }^{[2]}$. Finally, logistics service providers are used as suppliers. So far, the relevant domestic research has not been retrieved.

4.1.2. Collaborative management of supply chain. In supply chain management, blockchain technology can effectively reduce the transaction cost and complete the information sharing of the whole chain members, then ensure the performance of the supply chain. At present, scholars have carried out researches in the field of collaborative management of supply chain under the background of block chain.

In terms of supply chain management, Zhu Jianming et al. (2016) studied the application of blockchain technology and designed a simplified transaction structure for each transaction subject in the supply chain ${ }^{[13]}$. In the supply chain information resources, Wang Chuanlei et al. (2017) conducted research on the coupling relationship between blockchain and supply chain logistics information resources, and designed the supply chain logistics information ecosystem model based on block chain to promote the sustainable development of supply chain logistics information resource management ${ }^{[14]}$. In the supply chain trust management, Zhao Gongmin et al. (2019) proposed a supply chain trust management mechanism that includes the blockchain contract layer, consensus layer and data layer by applying block chain technology in the supply chain trust management system $^{[15]}$.

4.1.3. Food safety traceability. The emergence of blockchain provides a solution for food supply chain management, some scholars have launched relevant research on this topic. Blockchain is conducive to the traceability of food safety. Wang Puqing (2019) explored the root causes of food safety problems and discussed the role of blockchain technology in food safety management ${ }^{[16]}$. In terms of the design of food traceability system, Li Mingjia et al. (2018) studied the food safety traceability system using block chain technology and designed the scheme of using block chain technology in the food traceability system by analyzing the technical framework $^{[17]}$. Gao Shengqiao et al. (2020) built a dualchain storage optimization model of food supply chain data based on blockchain and verify that the program can effectively adapt to the supply chain and optimize storage resources through simulation experiments ${ }^{[18]}$.

\section{Research conclusions and prospects}

\subsection{Main research conclusions}

This paper uses Citespace software to conduct visualization analysis and data mining on 837 domestic 
literatures in the field of dual-chain fusion from 2015 to 2020, and analyses the research journals, authors, institutions, co-occurrence of key words and research hotspots. The main conclusions of this paper are as follows:

(1) Domestic journals in the field of dual-chain fusion have their own emphasis on theoretical basis, theme and content, empirical methods, and practical application. Among them, China Finance, Southwest Finance, and Journal of Commercial Economics focus on the practical application of blockchain in supply chain related fields. China Business and Market, and Construction Economy focus on empirical research. Computer Engineering and Applications, and Computer Engineering focus on the development of related theories and technologies.

(2) Scholars such as Song Hua and $\mathrm{Xu}$ Peng are leading scholars in the field. And domestic research in the dual-chain fusion field is in a state of high diffusion degree and low concentration degree, and there are obvious differences in disciplines between research teams, and scholars lack close contact and sufficient communication, so they are in a relatively independent research state.

(3) Research institutions in field of dual-chain fusion are mainly concentrated in the School of Economics and Management, Law School, and School of Information Management of universities. And these research institutions are relatively concentrated, but the cooperation among institutions is lacking.

(4) The research hotspots of Chinese scholars in the field in the past five years mainly focused on application scenarios, technological development, application entities, and funding. And this paper further summarizes the hot research topics in the field of dual-chain fusion: supply chain finance, supply chain collaborative management and food traceability.

\subsection{Shortage of research}

This paper only selects literatures from "CSSCI Journals", "CSCD Journals" and "Peking University Core Journals" as the research object, which may omit some research result. Second, due to the huge amount of data, this paper can only study and analyse from the macro and overall perspective, and it is difficult to analyse more detailed fields. Therefore, in the following research, we will continue to optimize data sources and data screening standards to further improve the quality of research.

\subsection{Future research outlook}

Double-chain fusion has been extended to finance, security, product traceability and other aspects, and has a huge impact on the supply chain. Therefore, hot issues in this field still need to be further explored and analysed.

(1) The composition of the supply chain contains different subjects. In the continuous exchange and cooperation between these subjects, the trust problem is inevitable. However, the current domestic research on the trust problem of the supply chain is relatively lacking. In the future, we can explore the integration of blockchain technology with other technology to improve the transparency of companies in the supply chain through the immutability of blockchain, and deal with the lack of trust in the supply chain at the technical level.

(2) We can analyse the solution to the problem of low efficiency of core enterprises in the field of supply chain finance based on block chain technology, so as to provide a strong guarantee for the interests of enterprises in the supply chain.

(3) The current application scenarios of blockchain technology are mainly food, agricultural products, and fresh supply chain fields. In the future, blockchain can be applied to more scenarios, such as supply chain traceability systems in other scenarios, including drug traceability, steel traceability, wood Traceability, traceability of automobile parts and so on.

(4) Double-chain integration can effectively solve the problem of information opacity in the supply chain, but at the same time it will also increase the risk of some fraud and crime. In the future, based on the characteristics of blockchain, a regulatory system can be established in the supply chain companies to supervise the supply chain systematically and comprehensively, and effectively avoid the risk of fraud and crime.

\section{Funding Resources}

This paper was supported by the Department of Science and Technology of Sichuan Province Fund (2021JDRC0002).

\section{References}

1. Zhao K et al. (2017) A Review of Supply Chain Management in China in Recent Ten Years-Based on Word Frequency Analysis (in Chinese). Modern Business Trade Industry, 30: 37-39.

2. Xue Y X. (2020) Hotspots and Trend of Supply Chain Management Based on CitesSpace (in Chinese). Logistics Engineering and Management, 42: 102-105.

3. Zhai T, Li W L. (2019) Research Focus and Current Situation of Blockchain Industry in China- Based on Analysis of Chinese Patent Documents (in Chinese). China Invention \& Patent, 16: 49-54.

4. Wang J Y, Wu Q L, Cao H Y. (2019) Overview of Research on Typical Application of Domestic Block Chain (in Chinese). Science \& Technology and Economy, 32: 1-6.

5. Li X D. (2019) The Research Review of "Blockchain + Logistics" (in Chinese). Journal of Guangdong Communication Polytechnic, 18: 42-47.

6. Yi Q, Yang H M, Wang K X. (2020) Review of Researches on Blockchain Technology Application in Logistics Field (in Chinese). Logistics Technology, 39: 129-133.

7. Shi J Z, Guo J E. (2015) Study on the Development and Domestic Practice of Supply Chain Finance from the Perspective of the Internet (in Chinese). Journal of Xi'an Jiaotong University (Social Sciences), 35: 10-16. 
8. Zhuang L, Zhao Y F. (2019) Research on application mode and development path of block chain technology (in Chinese). Journal of Finance and Economics, 40: 33-38.

9. Deng A M, Li Y F. (2019) "Intelligent Factoring" Business Model and Game Analysis in the Supply Chain Based on Block Chain (in Chinese). Management Review, 31: 231-240.

10. Chen Y H, Meng Z L, Zeng Y. (2018) Trade Credit Loan Pricing and Supply Chain Finance Pattern Choice Based on Retailers' Heterogeneity (in Chinese). Systems Engineering-Theory \& Practice, 38: 2479-2490.

11. Tu J P, Yang X. (2013) Research on Performance Evaluation of Supply Chain Financing Model Based on E-commerce Platform (in Chinese). Management World, 29: 182-183.

12. Wang M Z, Zhou L, Liu W W. (2017) Revenuesharing Trade Credit Contract for Coordinating Multiple Competing Retailers When Incorporating Default Risk (in Chinese). Operations Research and Management Science, 26: 1-11.

13. Zhu J M, Fu Y G. (2016) Supply Chain Dynamic Multi-center Coordination Authentication Model
Based on Block Chain (in Chinese). Chinese Journal of Network and Information Security, 2: 00019-100019-7.

14. Wang C L et al. (2017) A Model of Logistics Information Ecosphere of Supply Chain Based on Block Chain (in Chinese). Information Studies: Theory \& Application, 40: 115-121.

15. Zhao G M et al. (2019) Study on the Trust Management Mechanism of Supply Chain Based on Blockchain (in Chinese). Credit Reference, 37: 2531 .

16. Wang P Q et al. (2019) Application of Blockchain Technology in Food Safety Management (in Chinese). Journal of Agrotechnical Economics 38: 82-90.

17. Li M J. (2019) Food Safety Tracing Technology Based on Block Chain (in Chinese). Food Science, 40: 279-285.

18. Gao S Q, Liu X L, Gao Y P. (2020) Optimized Model of Dual-chain Storage of Food Supply Chain Data Based on Blockchain (in Chinese). Food \& Machinery, 36: 63-74. 